\title{
Relativistic screened hydrogenic radial integrals
}

\author{
F.H. Ruano , J.G. Rubiano ，M.A. Mendoza , J.M. Gil , R. Rodríguez \\ R. Florido , P. Martel , E. Mínguez
}

\begin{abstract}
A B S T R A C T
The computation of dipole matrix elements plays an important role in the study of absorption or emission of radiation by atoms in several fields such as astrophysics or inertial confinement fusion. In this work we obtain closed formulas for the dipole matrix elements of multielectron ions suitable for using in the framework of a Relativistic Screened Hydrogenic Model.
\end{abstract}

\section{Introduction}

The computation of dipole matrix elements plays an important role in the study of absorption or emission of radiation by atoms in several fields such as astrophysics, inertial confinement fusion and laser applications, among others. The dipole oscillator strengths which characterize the radiative transitions between two bound states are a key piece in the simulation of the absorption or emission spectra of ions and these quantities are proportional to the square of the radial dipole matrix elements. Thus they have been the object of an intensive investigation to derive analytical or numerical procedures to compute them into a non-relativistic [1-10] or a relativistic [11-16] framework. Many of the calculations leading to obtain closed analytical expressions for the matrix elements of multielectron atoms are based on different approaches of the Quantum Defect Theory (QDT) in combination, or not, with the WKB method [8,17]. Such models obtain analytical solutions of the Schrödinger or
Dirac equation including the non-hydrogenic contributions by simply replacing the hydrogenic radial solutions by similar ones depending on effective quantum numbers and an effective charge. A detailed review of these models is shown in the papers of Nana Engo et al. [16,18], who carried out a comprehensive study of different quasirelativistic and fully relativistic approaches based on the quantum defect model, providing closed-form expressions for the calculation of relativistic matrix elements.

In the field of laser-plasma interaction research, it is a common approach to use the screened hydrogenic model (SHM) to compute the properties of multielectron atoms. This model calculates the energy levels of the multielectron atom by using the analytical expressions of the hydrogenic atom computed with appropriate screened nuclear charges [19-25]. The SHM used in the average ion model context provides electronic populations with good accuracy and it also allows computing the opacity, the emissivity and the equation of state of plasma easily. For this reason the model has been widely used in the simulation of inertial confinement fusion [26-35]. In this model, the multielectron atom is described by singleelectron hydrogenic wave function depending on an effective charge characteristic of any quantum state 
[36]. Since the dipole matrix elements depend only on the wave function of the ions, it is necessary to compute them using different screened nuclear charges $Q_{k}$ and $Q_{K}$ in order to describe the initial and final state of the running electron. Unlike the quantum defect models cited above, the SHM approach is simpler, because all non-hydrogenic contributions are concentrated only in the screened charge. So the expressions derived in the context of QDT are not directly applicable to the SHM since the screened charges of the SHM are not equivalent to the effective charges of the QDT. Few authors provide general formulas for dipole matrix elements suitable for the Screened Hydrogenic Model approach and mainly they do it in the non-relativistic context [37-39] so there is a lack of study in the relativistic frame.

In this paper we obtain closed formulas for the dipole matrix elements of multielectron ions expressed in terms of elementary functions depending on the characteristic screened charges, so that they are suitable for their use in the Relativistic Screened Hydrogenic Model.

\section{Background}

\subsection{Relativistic screened hydrogenic model}

In 1930, Slater [25] was the first to introduce the concept of screening constant to calculate the energies of multielectron atoms, although he gave only empirical rules to estimate the screening parameters. Subsequently, Mayer [21] developed a model to compute opacities in plasmas at high density and temperature in which the concept of average atom was introduced and the SHM was developed, being the first author to compute several sets of universal screening constants.

The theoretical framework of SHM is due to Layzer in the late 1950s [20], who developed a theory based on the concept of screening to determine atomic spectra. In this theory, each atomic state is characterized by a set of screening parameters instead of a wave function. More [23] developed a new SHM and introduced a new set of screening constants obtained from the fit to a database with 800 ionization potentials of 30 elements, calculated using a self-consistent Hartree-Fock-Slater code. The one-electron states obtained with this set of screening constants are degenerated with respect to angular momentum, i.e., each state was labeled by the principal quantum number $n$. Faussurier et al. [19] reviewed in detail the theoretical basis of SHM and provided a whole new set of coefficients with $l$-splitting, obtained from the fit to a database with about 15,600 values of Hartree-Fock excitation energies and ionization potentials. This model gives more accurate results than More's model.

Rubiano et al. [24] developed a method that do not use screening constants for calculating the screened charge and obtaining the screened charges and the external screening of the SHM from analytical potentials. Using this method and analytical screened hydrogenic wave functions, they proposed a SHM model with $n l j$ splitting which was strictly valid for the ground states of He-like to U-like ions. Recently, we have developed a New Relativistic Screened Hydrogenic Model (NRSHM) based on a new set of universal screening constants [22], obtained by fitting to a database containing 61,356 high quality atomic data. It was conceived to deal with ground and excited configurations of medium and highly ionized atoms and in this paper we will make use of it to compute the screened charges $Q_{k}$ and $Q_{k}$.

\subsection{The Dirac equation and the relativistic screened hydrogenic model}

In the screened hydrogenic model each bound electron moves in the individual potential

$U_{n l j}(r)=-\frac{Q_{n l j}}{r}+A_{n l j}$,

depending on the nuclear charge and the other atomic electrons. In this equation the quantum state of the bound electron is described in a relativistic frame by the quantum numbers $n, l$, and $j$, the parameter $Q_{n j}$ is called the screened charge of the level and $A_{n l j}$ is a constant term depending on the screened charge which will be referred further as the external screening constant. Several methods can be found in the literature to determine these parameters $[24,38]$.

The Dirac equation has analytical solutions for this potential and therefore the wave functions and energy levels can be expressed in an analytical form. The exact solutions of the stationary Dirac equation $[40,41]$

$H \psi=\left(c \alpha \mathbf{p}+m c^{2} \beta-\frac{Q_{n l j}}{r}+A_{n l j}\right) \psi=\epsilon_{n l j} \psi$

are

$\psi=\left(\begin{array}{c}\varphi \\ \chi\end{array}\right)=\left(\begin{array}{c}\mathcal{Y}_{j m}^{ \pm}(\mathbf{n}) f_{n l j}(r) \\ i \mathcal{Y}_{j m}^{\mp}(\mathbf{n}) g_{n l j}(r)\end{array}\right)$,

where the spinor spherical harmonics $\mathcal{Y}_{j m}^{ \pm}(\mathbf{n})=\mathcal{Y}_{j m}^{(j \pm 1 / 2)}(\mathbf{n})$ are given explicitly in terms of the ordinary spherical functions $Y_{l m}(\mathbf{n}), \mathbf{n}=\mathbf{n}(\theta, \phi)=\mathbf{r} / r$, and the special ClebschGordan coefficients with the spin $1 / 2$ as follows:

$\mathcal{Y}_{j m}^{ \pm}(\mathbf{n})=\left(\begin{array}{c}\mp \sqrt{\frac{j+1 / 2) \mp(m-1 / 2)}{2 j+(1 \pm 1)}} Y_{j \pm 1 / 2, m-1 / 2}(\mathbf{n}) \\ \sqrt{\frac{(j+1 / 2) \pm(m+1 / 2)}{2 j+(1 \pm 1)}} Y_{j \pm 1 / 2, m+1 / 2}(\mathbf{n})\end{array}\right)$,

where $j$ stands for the total angular momentum and $m$ is its projection. The large component $F_{n l j}(r)$ and the small component $G_{n l j}(r)$ of the radial wave function are, in atomic units [38]

$$
\begin{aligned}
F_{n l j}(r)= & r f_{n l j}(r) \\
= & \frac{B_{n l j}}{4 \tilde{a} v(\kappa-v) \alpha^{1 / 2}} x^{v} e^{-x / 2}\left[f_{1} x L_{n-j-3 / 2}^{2 v+1}(x)+f_{2} L_{n-j-1 / 2}^{2 v-1}(x)\right], \\
G_{n l j}(r)= & r g_{n l j}(r)=\frac{B_{n l j}}{4 a \tilde{v}(\kappa-v) \alpha^{1 / 2}} x^{v} e^{-x / 2} \\
& \times\left[g_{1} x L_{n-j-3 / 2}^{2 v+1}(x)+g_{2} L_{n-j-1 / 2}^{2 v-1}(x)\right] .
\end{aligned}
$$

They are expressed in terms of the generalized Laguerre polynomials $L_{\alpha}^{\beta}(x)$ and depending on the variable $x=2 \tilde{a} r / \alpha$. In this expression $\alpha$ is the fine structure constant, $r$ is the radial coordinate and $\tilde{a}$ is a parameter that depends on the screened charge as well as the 
quantum numbers of the state, and it is defined using the following expression:

$\tilde{a}=\frac{\alpha Q_{n l j}}{\sqrt{\left(n-j-\frac{1}{2}+v\right)^{2}+\left(\alpha Q_{n l j}\right)^{2}}}$,

where $v=\sqrt{\left(j+\frac{1}{2}\right)^{2}-\left(\alpha Q_{n l j}\right)^{2}}$ and $\kappa=-2(j-l)\left(j+\frac{1}{2}\right)$. The rest of the terms in Eq. (5) are given by

$f_{1}=\frac{\tilde{a}\left(\alpha Q_{n l j}\right)^{2}}{\tilde{a} \kappa\left(n-j-\frac{1}{2}+v\right)-\alpha Q_{n l j} v}, \quad f_{2}=\kappa-v$,

$g_{1}=\frac{f_{1} f_{2}}{\alpha Q_{n l j}}, \quad g_{2}=\alpha Q_{n l j}$

Finally, the normalization constant has the following expression:

$B_{n l j}=\frac{2 \tilde{a}^{2}}{\alpha Q_{n l j}} \sqrt{\frac{f_{2}\left(n-j-\frac{1}{2}\right) !\left[\tilde{a} \kappa\left(n-j-\frac{1}{2}+v\right)-\alpha Q_{n l j} v\right]}{\Gamma\left(n-j-\frac{1}{2}+2 v\right)}}$

In Eq. (5) it is necessary to assign $L_{-1}^{2 v+1}=0$ for $j=n-1 / 2$. The wave functions defined in this way depend on the screened charges of each orbital $Q_{n l j}$, and they are independent on the external screening constant $A_{n l j}$, which modifies only the energy levels $\epsilon_{n l j}$. They are given by the expression:

$\epsilon_{n l j}=m_{e} c^{2}\left[\left(1+\left[\frac{\alpha Q_{n l j}}{n-j-1 / 2+\sqrt{(j+1 / 2)^{2}-\left(\alpha Q_{n l j}\right)^{2}}}\right]^{2}\right)^{-1 / 2}-1\right]+A_{n l j}$,

where $m_{e}$ is the electron mass and $c$ is the speed of light.

\section{Semi-relativistic calculation of matrix elements}

Dipole radial matrix elements are required to compute oscillator strengths needed to model radiative transitions between bound states. These matrix elements are given by $[42]$

$$
\begin{aligned}
& \left\langle n, l, j|r| n^{\prime}, l^{\prime}, j^{\prime}\right\rangle=\int_{0}^{\infty}\left[F_{n l j}(r) F_{n^{\prime} l^{\prime} j^{\prime}}(r)+G_{n l j}(r) G_{n^{\prime} j^{\prime}}(r)\right] r d r \\
& \quad=\int_{0}^{\infty} F_{n l j}(r) r F_{n l^{\prime} j^{\prime}}(r) d r+\int_{0}^{\infty} G_{n l j}(r) r G_{n^{\prime} l^{\prime} j^{\prime}}(r) d r=r_{F}+r_{G} .
\end{aligned}
$$

In the following paragraphs we will make explicit the dependence on the quantum numbers of the parameters of the radial wave functions defined in the previous section by means of the subindex $k=n l j$ and $k^{\prime}=n^{\prime} l^{\prime} j^{\prime}$. Also, we will explicitly show the dependence on the radial coordinate $r$ instead of the variable $x$ defined above. Radial wave functions are now written as

$$
\begin{aligned}
& F_{k}(r)=C_{k} a_{k}^{v_{k}} r^{v_{k}} e^{-a_{k} r}\left[f_{1 k} 2 a_{k} r L_{m}^{2 v_{k}+1}\left(2 a_{k} r\right)+f_{2 k} L_{m+1}^{2 v_{k}-1}\left(2 a_{k} r\right)\right], \\
& G_{k}(r)=C_{k} a_{k}^{v_{k}} r^{v_{k}} e^{-a_{k} r}\left[g_{1 k} 2 a_{k} r L_{m}^{2 v_{k}+1}\left(2 a_{k} r\right)+g_{2 k} L_{m+1}^{2 v_{k}-1}\left(2 a_{k} r\right)\right],
\end{aligned}
$$

where we have also defined the following state depending constants:

$C_{k}=\frac{2^{v_{k}-2} B_{k}}{\tilde{a} v_{k}\left(\kappa_{k}-v_{k}\right) \alpha^{1 / 2}}, \quad a_{k}=\tilde{a} / \alpha, \quad m=n-j-\frac{3}{2}$.

If we write out the term $r_{F}$ in Eq. (11), we obtain

$$
\begin{aligned}
r_{F}= & \int_{0}^{\infty} F_{k} r F_{k} d r=\int_{0}^{\infty} C_{k} C_{k} a_{k}^{v_{k}} a_{k^{\prime}}^{v^{\prime}} r^{v_{k}+v_{k^{\prime}}+1} e^{-\left(a_{k}+a_{k^{\prime}}\right) r} \\
& \times\left[f_{1 k^{2}} 2 a_{k} r L_{m}^{2 v_{k}+1}\left(2 a_{k} r\right)+f_{2 k} L_{m+1}^{2 v_{k}-1}\left(2 a_{k} r\right)\right] \\
& \times\left[f_{1 k^{\prime}} 2 a_{k^{\prime}} r L_{m^{\prime}}^{2 v^{\prime}+1}\left(2 a_{k^{\prime}} r\right)+f_{2 k^{\prime}} L_{m^{\prime}+1}^{2 v_{k^{\prime}}-1}\left(2 a_{k^{\prime}} r\right)\right] d r,
\end{aligned}
$$

this yields

$$
\begin{aligned}
& r_{F}=4 C_{k} C_{k^{\prime}} a_{k}^{v_{k}+1} a_{k^{\prime}}^{v_{k^{\prime}+1}} f_{1 k} f_{1 k} \\
& \times \int_{0}^{\infty} r^{v_{k}+v_{k^{\prime}}+3} e^{-\left(a_{k}+a_{k^{\prime}}\right) r} L_{m}^{2 v_{k}+1}\left(2 a_{k} r\right) L_{m^{\prime}}^{2 v_{k^{\prime}}+1}\left(2 a_{k^{\prime}} r\right) d r \\
& +2 C_{k} C_{k^{\prime}} a_{k}^{v_{k}+1} a_{k^{\prime}}^{v_{k^{\prime}}} f_{1 k} f_{2 k^{\prime}} \\
& \times \int_{0}^{\infty} r^{v_{k}+v_{k^{\prime}}+2} e^{-\left(a_{k}+a_{k^{\prime}}\right) r} L_{m}^{2 v_{k}+1}\left(2 a_{k} r\right) L_{m^{\prime}+1}^{2 v_{k^{\prime}}-1}\left(2 a_{k^{\prime}} r\right) d r \\
& +2 C_{k} C_{k^{\prime}} a_{k}^{v_{k}} a_{k^{\prime}}^{v_{k^{\prime}}+1} f_{2 k} f_{1 k^{\prime}} \\
& \times \int_{0}^{\infty} r^{v_{k}+v_{k^{\prime}}+2} e^{-\left(a_{k}+a_{k^{\prime}}\right) r} L_{m+1}^{2 v_{k}-1}\left(2 a_{k} r\right) 2 a_{k^{\prime}} L_{m^{\prime}}^{2 v_{k^{\prime}}+1}\left(2 a_{k^{\prime}} r\right) d r \\
& +C_{k} C_{k^{\prime}} a_{k}^{v_{k}} a_{k^{\prime}}^{v_{k}} f_{2 k} f_{2 k^{\prime}} \\
& \times \int_{0}^{\infty} r^{v_{k}+v_{k^{\prime}}+1} e^{-\left(a_{k}+a_{k^{\prime}}\right) r} L_{m+1}^{2 v_{k}-1}\left(2 a_{k} r\right) L_{m^{\prime}+1}^{2 v_{k^{\prime}}-1}\left(2 a_{k} r\right) d r,
\end{aligned}
$$

and, finally, it can be written as

$$
\begin{aligned}
r_{F}= & 4 C_{k} C_{k^{\prime}} a_{k}^{v_{k}+1} a_{k^{\prime}}^{v_{k^{\prime}+1}} f_{1 k} f_{1 k^{\prime}} I_{2}\left(m, 2 v_{k}+1, m^{\prime}, 2 v_{k}+1\right) \\
& +2 C_{k} C_{k^{\prime}} a_{k}^{v_{k}+1} a_{k^{\prime}}^{v_{k}} f_{1 k} f_{2 k^{\prime}} I_{1}\left(m, 2 v_{k}+1, m^{\prime}+1,2 v_{k^{\prime}}-1\right) \\
& +2 C_{k} C_{k^{\prime}} a_{k}^{v_{k}} a_{k^{\prime}}^{v_{k^{\prime}}+1} f_{2 k} f_{1 k^{\prime}} I_{1}\left(m+1,2 v_{k}-1, m^{\prime}, 2 v_{k^{\prime}}+1\right) \\
& +C_{k} C_{k^{\prime}} a_{k}^{v_{k}} a_{k^{\prime}}^{v_{k}} f_{2 k} f_{2 k^{\prime}} I_{0}\left(m+1,2 v_{k}-1, m^{\prime}+1,2 v_{k^{\prime}}-1\right),
\end{aligned}
$$

where it has been defined as the function

$$
\begin{aligned}
& I_{\alpha}\left(q_{k}, p_{k}, q_{k^{\prime}}, p_{k^{\prime}}\right) \\
& \quad=\int_{0}^{\infty} r^{\left(v_{k}+v_{k^{\prime}}+1\right)+\alpha} e^{-\left(a_{k}+a_{k^{\prime}}\right) r} L_{q_{k}}^{p_{k^{\prime}}}\left(2 a_{k} r\right) L_{q_{k^{\prime}}}^{p_{\chi}}\left(2 a_{k^{\prime}} r\right) d r,
\end{aligned}
$$

which will be further referred as RR-integral. Using this notation the term associated to the minor component $r_{G}$ can be expressed in a similar way

$$
\begin{aligned}
r_{G}= & 4 C_{k} C_{k^{\prime}} a_{k}^{v_{k}+1} a_{k^{\prime}}^{v_{k^{\prime}+1}} g_{1 k} g_{1 k^{\prime}} I_{2}\left(m, 2 v_{k}+1, m^{\prime}, 2 v_{k^{\prime}}+1\right) \\
& +2 C_{k} C_{k^{\prime}} a_{k}^{v_{k}+1} a_{k^{\prime}}^{v_{k^{\prime}}} g_{1 k} g_{2 k^{\prime}} I_{1}\left(m, 2 v_{k}+1, m^{\prime}+1,2 v_{k^{\prime}}-1\right) \\
& +2 C_{k} C_{k^{\prime}} a_{k}^{v_{k}} a_{k^{\prime}}^{v_{k^{\prime}}+1} g_{2 k} g_{1 k^{\prime}} I_{1}\left(m+1,2 v_{k}-1, m^{\prime}, 2 v_{k^{\prime}}+1\right) \\
& +C_{k} C_{k^{\prime}} a_{k}^{v_{k}} a_{k^{\prime}}^{v^{\prime}} g_{2 k} g_{2 k^{\prime}} I_{0}\left(m+1,2 v_{k}-1, m^{\prime}+1,2 v_{k^{\prime}}-1\right) .
\end{aligned}
$$

Finally, joining all these results we obtain for the matrix elements the expression

$$
\begin{aligned}
& \left\langle k|r| k^{\prime}\right\rangle=4 C_{k} C_{k} a_{k}^{v_{k}+1} a_{k^{\prime}+1}^{v_{V^{\prime}+1}}\left(f_{1 k} f_{1 K}+g_{1 k} g_{1 K}\right) I_{2}\left(m, 2 v_{k}+1, m^{\prime}, 2 v_{k^{\prime}}+1\right) \\
& +2 C_{k} C_{k} a_{k}^{v_{k}+1} a_{k^{\prime}}^{v_{k}}\left(f_{1 k} f_{2 K}+g_{1 k} g_{2 k}\right) I_{1}\left(m, 2 v_{k}+1, m^{\prime}+1,2 v_{K}-1\right) \\
& +2 C_{k} C_{k} a_{k}^{v_{k}} a_{k^{\prime}}^{y_{k^{\prime}}+1}\left(f_{2 k} f_{1 K}+g_{2 k} g_{1 k^{\prime}}\right) I_{1}\left(m+1,2 v_{k}-1, m^{\prime}, 2 v_{k^{\prime}}+1\right) \\
& +C_{k} C_{k^{\prime}} a_{k}^{v_{k}} a_{K^{\prime}}^{v_{K^{\prime}}}\left(f_{2 k} f_{2 K^{\prime}}+g_{2 k} g_{2 k^{\prime}}\right) I_{0}\left(m+1,2 v_{k}-1, m^{\prime}+1,2 v_{k^{\prime}}-1\right),
\end{aligned}
$$


which depends on integrals like the ones defined in Eq. (14). In the following section we develop a method to obtain an analytical expression for these integrals.

\section{Analytical solution of the RR-Integrals}

The RR-integral in Eq. (14) has the general form:

$$
\begin{aligned}
& I_{s}\left(q_{k}, p_{k}, q_{k^{\prime}}, p_{k^{\prime}}\right)=\int_{0}^{\infty} r^{s} e^{-\left(a_{k}+a_{k^{\prime}}\right) r} L_{q_{k}}^{p_{k}}\left(2 a_{k} r\right) L_{q_{k^{\prime}}}^{p_{\chi^{\prime}}}\left(2 a_{k^{\prime}} r\right) d r, \\
& a_{k}+a_{k^{\prime}}>0, s>0 .
\end{aligned}
$$

In order to obtain an analytical solution for this integral we first substitute one of the associated Laguerre polynomial by its power series expansion

$L_{q_{k}}^{p_{k}}\left(2 a_{k} r\right)= \begin{cases}\sum_{t=0}^{p_{k}} \frac{\left(-2 a_{k} r\right)^{t} \Gamma\left(q_{k}+p_{k}+1\right)}{\Gamma(t+1) \Gamma\left(t+p_{k}+1\right) \Gamma\left(q_{k}-t+1\right)} & \text { if } q_{k} \geq 0, \\ 0 & \text { if } q_{k}<0,\end{cases}$

and rearranging this yields

$$
\begin{aligned}
I_{s}\left(q_{k}, p_{k}, q_{k^{\prime}}, p_{k^{\prime}}\right)= & \sum_{t=0}^{p_{k}} \frac{\left(-2 a_{k}\right)^{t} \Gamma\left(q_{k}+p_{k}+1\right)}{\Gamma(t+1) \Gamma\left(t+p_{k}+1\right) \Gamma\left(q_{k}-t+1\right)} \\
& \times \int_{0}^{\infty} r^{s+t} e^{-\left(a_{k}+a_{k^{\prime}}\right) r} L_{q_{k^{\prime}}}^{p_{k^{\prime}}}\left(2 a_{k^{\prime}} r\right) d r
\end{aligned}
$$

The integral in the above expression has the next analytical solution depending on a Gauss hypergeometric function [43]

$$
\begin{aligned}
& \int_{0}^{\infty} x^{\beta} e^{-z x} L_{n}^{\alpha}(x) d x \\
& \quad=\frac{\Gamma(\beta+1) \Gamma(\alpha+n+1)}{n ! \Gamma(\alpha+1)} z^{-\beta-1}{ }_{2} F_{1}(-n, \beta+1, \alpha+1,1 / z)
\end{aligned}
$$

$\operatorname{Re} \beta>-1, \operatorname{Re} z>0$.

By substituting $x=2 a_{k^{\prime}} r$ into Eq. (19)

$$
\begin{aligned}
I_{s}\left(q_{k}, p_{k}, q_{k^{\prime}}, p_{k^{\prime}}\right) & =\sum_{t=0}^{p_{k}} \frac{\left(-2 a_{k}\right)^{t} \Gamma\left(q_{k}+p_{k}+1\right)}{\left(2 a_{k^{\prime}}\right)^{s+t+1} \Gamma(t+1) \Gamma\left(t+p_{k}+1\right) \Gamma\left(q_{k}-t+1\right)} \\
& \times \int_{0}^{\infty} x^{s+t} e^{-\left(\left(a_{k}+a_{k^{\prime}}\right) / 2 a_{k^{\prime}}\right) x} L_{q_{k^{\prime}}}^{p_{k^{\prime}}}(x) d x,
\end{aligned}
$$

and taking into account that $z=\left(a_{k}+a_{k}^{\prime}\right) / 2 a_{k^{\prime}}$ we finally obtain the expression

$$
\begin{aligned}
& I_{s}\left(q_{k}, p_{k}, q_{k^{\prime}}, p_{k^{\prime}}\right)=\sum_{t=0}^{p_{k}} \frac{\left(-2 a_{k}\right)^{t} \Gamma\left(q_{k}+p_{k}+1\right)}{\left(2 a_{k^{\prime}}\right)^{s+t+1} \Gamma(t+1) \Gamma\left(t+p_{k}+1\right) \Gamma\left(q_{k}-t+1\right)} \\
& \times \frac{\Gamma(s+t+1) \Gamma\left(p_{k^{\prime}}+q_{k^{\prime}}+1\right)}{q_{k^{\prime}} ! \Gamma\left(p_{k}^{\prime}+1\right)} z^{-(s+t+1)}{ }_{2} F_{1}\left(-q_{k^{\prime}}, s+t+1, p_{k^{\prime}}+1,1 / z\right) .
\end{aligned}
$$

An alternative solution of the RR-integrals that provides an analytical formula easy to implement into a computational code is shown in Appendix A.

\section{Fully relativistic calculation of matrix elements}

\subsection{General case}

The semi-relativistic approximation shown in Section 3 is appropriated while evaluating the relativistic changes only due to wave functions. To obtain a better evaluation of the impact of the relativistic operator on radial integrals and therefore on oscillator strengths a fully relativistic calculation is needed to take into account the effect of retardation. This effect takes into account the delay in the electromagnetic interaction between bound electron and the incident photon due to the finite value of the speed of light. We included it by computing the matrix elements in the Babushkin gauge that reduces to the length form in the non-relativistic limit. In this context the matrix elements adopts the form [42]

$r_{k k^{\prime}} \rightarrow \frac{3}{k_{\lambda}}\left[J_{1}+\frac{\Delta \kappa}{2} I_{2}^{+}+I_{2}^{-}\right]$,

$k_{\lambda}$ being the wave number of the photon, $\Delta \kappa=\kappa_{k}-\kappa_{k}$, with $\kappa_{k}=-2\left(j_{k}-l_{k}\right)\left(j_{k}+1 / 2\right)$ and

$I_{2}^{ \pm}=\int_{0}^{\infty}\left[F_{k}(r) G_{k^{\prime}}(r) \pm G_{k}(r) F_{k^{\prime}}(r)\right] j_{2}\left(k_{\lambda} r\right) d r$

$J_{1}=\int_{0}^{\infty}\left[F_{k}(r) F_{K^{\prime}}(r)+G_{k}(r) G_{K^{\prime}}(r)\right] j_{1}\left(k_{\lambda} r\right) d r$.

In these equations $F_{k}(r)$ and $G_{k}(r)$ are the large and small component of the radial wave function (5), respectively, and the function $j_{L}\left(k_{\lambda} r\right)$ is the spherical Bessel function of order $L$

$j_{L}\left(k_{\lambda} r\right)=\left(k_{\lambda} r\right)^{L} \sum_{p=0}^{\infty}\left(-\frac{1}{2}\right)^{p} \frac{\left(k_{\lambda} r\right)^{2 p}}{p !(2 L+2 p+1) ! !}$.

where !! denotes the double factorial. An analytical solution for the matrix elements $r_{k k^{\prime}}$, in terms of the RR-integral, is obtained by substituting Eqs. (24)-(26)) into Eq. (23)

$$
\begin{aligned}
r_{k k}= & 3 \sum_{p=0}^{N}\left(-\frac{1}{2}\right)^{p} \frac{k_{\lambda}^{2 p}}{p !(2 p+3) ! !} \\
& \times \int_{0}^{\infty}\left[F_{k}(r) F_{k^{\prime}}(r)+G_{k}(r) G_{k^{\prime}}(r)\right] r^{2 p+1} d r \\
& +3\left(\frac{\Delta \kappa}{2}+1\right) \sum_{p=0}^{N}\left(-\frac{1}{2}\right)^{p} \frac{k_{\lambda}^{2 p+1}}{p !(2 p+5) ! !} \\
& \times \int_{0}^{\infty} F_{k}(r) G_{k^{\prime}}(r) r^{2 p+2} d r \\
& +3\left(\frac{\Delta \kappa}{2}-1\right) \sum_{p=0}^{N}\left(-\frac{1}{2}\right)^{p} \frac{k_{\lambda}^{2 p+1}}{p !(2 p+5) ! !} \\
& \times \int_{0}^{\infty} G_{k}(r) F_{k^{\prime}}(r) r^{2 p+2} d r .
\end{aligned}
$$

The integrals in the above expression can be expressed as a sum of four terms containing RR-integrals, $I_{\gamma}(n, \alpha, m, \beta)$, where the values of the order $\alpha$ of Eq. (14) are between $2 p+2$ and $2 p$ for the first one, and $2 p+3$ and $2 p+1$ for the two latter

$$
\begin{aligned}
\int_{0}^{\infty} & {\left[F_{k}(r) F_{k^{\prime}}(r)+G_{k}(r) G_{k^{\prime}}(r)\right] r^{2 p+1} d r } \\
= & \mathcal{A}\left(f_{1 k} f_{1 k^{\prime}}+g_{1 k^{\prime}} g_{1 k^{\prime}}\right) I_{2 p+2}\left(m, 2 v_{k}+1, m^{\prime}, 2 v_{k^{\prime}}+1\right) \\
& +\mathcal{B}\left(f_{1 k} f_{2 k^{\prime}}+g_{1 k} g_{2 k^{\prime}}\right) I_{2 p+1}\left(m, 2 v_{k}+1, m^{\prime}+1,2 v_{k^{\prime}}-1\right) \\
& +\mathcal{C}\left(f_{2 k} f_{1 k^{\prime}}+g_{2 k} g_{1 k^{\prime}}\right) I_{2 p+1}\left(m+1,2 v_{k}-1, m^{\prime}, 2 v_{k^{\prime}}+1\right) \\
& +\mathcal{D}\left(f_{2 k} f_{2 k^{\prime}}+g_{2 k} g_{2 k^{\prime}}\right) I_{2 p}\left(m+1,2 v_{k}-1, m^{\prime}+1,2 v_{k^{\prime}}-1\right),
\end{aligned}
$$

$$
\begin{aligned}
& \int_{0}^{\infty} F_{k}(r) G_{k^{\prime}}(r) r^{2 p+2} d r=\mathcal{A} f_{1 k} g_{1 k^{\prime}} I_{2 p+3}\left(m, 2 v_{k}+1, m^{\prime}, 2 v_{k^{\prime}}+1\right) \\
& \quad+\mathcal{B} f_{1 k^{\prime}} g_{2 k^{\prime}} I_{2 p+2}\left(m, 2 v_{k}+1, m^{\prime}+1,2 v_{k^{\prime}}-1\right)
\end{aligned}
$$


Table 1

Matrix elements (a.u.) computed for some ions of the Ne isoelectronic sequence. $\Delta r_{k k}$ is the difference between values obtained from the analytical expression and values computed from the wave function integration.

\begin{tabular}{|c|c|c|c|c|c|}
\hline Ion & $k^{\prime}$ & $Q_{k^{\prime}}$ & $E_{k^{\prime}}$ & $r_{k k^{\prime}}$ & $\Delta r_{k k^{\prime}}$ \\
\hline $\mathrm{Ne}$ & $3 s_{1 / 2}$ & 4.1567 & -128.2287 & $-1.200 \times 10^{-1}$ & $4.163 \times 10^{-17}$ \\
\hline$Q_{k}=5.7108$ & $3 d_{3 / 2}$ & 1.3506 & -128.1450 & $6.853 \times 10^{-2}$ & 0.0000 \\
\hline \multirow[t]{5}{*}{$E_{k}=-128.6640$} & $3 d_{5 / 2}$ & 1.3235 & -128.1557 & $6.508 \times 10^{-2}$ & $1.388 \times 10^{-17}$ \\
\hline & $4 s_{1 / 2}$ & 2.7518 & -128.1445 & $-1.270 \times 10^{-1}$ & $5.274 \times 10^{-16}$ \\
\hline & $4 d_{3 / 2}$ & 1.2797 & -128.1372 & $4.457 \times 10^{-2}$ & $5.064 \times 10^{-13}$ \\
\hline & $4 d_{5 / 2}$ & 1.1933 & -128.1331 & $3.730 \times 10^{-2}$ & $1.388 \times 10^{-17}$ \\
\hline & $5 s_{1 / 2}$ & 1.8381 & -128.1722 & $-4.775 \times 10^{-2}$ & $1.943 \times 10^{-16}$ \\
\hline $\mathrm{Fe}^{+16}$ & $3 s_{1 / 2}$ & 20.1567 & -1121.7607 & $2.371 \times 10^{-2}$ & $2.012 \times 10^{-16}$ \\
\hline$Q_{k}=21.7108$ & $3 d_{3 / 2}$ & 17.3506 & -1118.5910 & $1.787 \times 10^{-1}$ & $2.776 \times 10^{-17}$ \\
\hline \multirow{5}{*}{$E_{k}=-1148.3810$} & $3 d_{5 / 2}$ & 17.3235 & -1118.5774 & $1.785 \times 10^{-1}$ & $1.110 \times 10^{-16}$ \\
\hline & $4 s_{1 / 2}$ & 18.7518 & -1112.3841 & $3.703 \times 10^{-3}$ & $4.124 \times 10^{-16}$ \\
\hline & $4 d_{3 / 2}$ & 17.2797 & -1111.3408 & $8.727 \times 10^{-2}$ & $1.249 \times 10^{-16}$ \\
\hline & $4 d_{5 / 2}$ & 17.1933 & -1111.2504 & $8.741 \times 10^{-2}$ & $8.327 \times 10^{-17}$ \\
\hline & $5 s_{1 / 2}$ & 17.8381 & -1108.4477 & $-6.506 \times 10^{-4}$ & $5.332 \times 10^{-16}$ \\
\hline $\mathrm{Au}^{+69}$ & $3 s_{1 / 2}$ & 73.1567 & -12441.9224 & $1.678 \times 10^{-2}$ & $4.857 \times 10^{-17}$ \\
\hline$Q_{k}=74.7108$ & $3 d_{3 / 2}$ & 70.3506 & -12411.7960 & $5.823 \times 10^{-2}$ & $3.469 \times 10^{-17}$ \\
\hline \multirow{5}{*}{$E_{k}=-12793.5433$} & $3 d_{5 / 2}$ & 70.3235 & -12407.5781 & $5.872 \times 10^{-2}$ & $6.939 \times 10^{-18}$ \\
\hline & $4 s_{1 / 2}$ & 71.7518 & -12299.8235 & $6.019 \times 10^{-3}$ & $1.258 \times 10^{-16}$ \\
\hline & $4 d_{3 / 2}$ & 70.2797 & -12288.7969 & $2.272 \times 10^{-2}$ & $3.469 \times 10^{-18}$ \\
\hline & $4 d_{5 / 2}$ & 70.1933 & -12286.6614 & $2.383 \times 10^{-2}$ & 0.0000 \\
\hline & $5 s_{1 / 2}$ & 70.8381 & -12236.9149 & $3.396 \times 10^{-3}$ & $7.156 \times 10^{-17}$ \\
\hline
\end{tabular}

$$
\begin{aligned}
& +\mathcal{C} f_{2 k} g_{1 k^{\prime}} I_{2 p+2}\left(m+1,2 v_{k}-1, m^{\prime}, 2 v_{k}+1\right) \\
& +\mathcal{D} f_{2 k} g_{2 k^{\prime}} I_{2 p+1}\left(m+1,2 v_{k}-1, m^{\prime}+1,2 v_{k}-1\right) \\
\int_{0}^{\infty} & G_{k}(r) F_{k^{\prime}}(r) r^{2 p+2} d r=\mathcal{A} g_{1 k} f_{1 k^{\prime}} I_{2 p+3}\left(m, 2 v_{k}+1, m^{\prime}, 2 v_{k^{\prime}}+1\right) \\
& +\mathcal{B} g_{1 k} f_{2 k^{\prime}} I_{2 p+2}\left(m, 2 v_{k}+1, m^{\prime}+1,2 v_{k^{\prime}}-1\right) \\
& +\mathcal{C} g_{2 k} f_{1 k^{\prime}} I_{2 p+2}\left(m+1,2 v_{k}-1, m^{\prime}, 2 v_{k}+1\right) \\
& +\mathcal{D} g_{2 k} f_{2 k^{\prime}} I_{2 p+1}\left(m+1,2 v_{k}-1, m^{\prime}+1,2 v_{k}-1\right)
\end{aligned}
$$

where

$\mathcal{A}=4 C_{k} C_{k} a_{k}^{v_{k}+1} a_{k^{\prime}}^{v_{k^{\prime}}+1}, \quad \mathcal{B}=2 C_{k} C_{k} a_{k}^{v_{k}+1} a_{k^{\prime}}^{v_{k^{\prime}}}$,

$\mathcal{C}=2 C_{k} C_{k} a_{k}^{v_{k}} a_{k^{\prime}}^{v_{k^{\prime}}+1}, \quad \mathcal{D}=C_{k} C_{k^{\prime}} a_{k}^{v_{k}} a_{k^{\prime}}^{v_{k^{\prime}}}$.

\subsection{Expression for small arguments of $j_{n}\left(k_{\lambda} r\right)$}

A closed expression for the matrix elements for $k_{\lambda} r \rightarrow 0$ can be obtained by retaining only the first term, $(p=0)$ in Eq. (26)

$j_{L}\left(k_{\lambda} r\right) \approx \frac{\left(k_{\lambda} r\right)^{L}}{(2 L+1) ! !}$,

and introducing it into Eqs. (24) and (25), this yields

$$
\begin{aligned}
r_{k k^{\prime}}= & \int_{0}^{\infty} F_{k}(r) F_{k^{\prime}}(r) r d r+\int_{0}^{\infty} G_{k}(r) G_{k^{\prime}}(r) r d r \\
& +\left[\frac{k_{\lambda}}{5}\left(\frac{\Delta \kappa}{2}+1\right)\right] \int_{0}^{\infty} F_{k}(r) G_{k^{\prime}}(r) r^{2} d r \\
& +\left[\frac{k_{\lambda}}{5}\left(\frac{\Delta \kappa}{2}-1\right)\right] \int_{0}^{\infty} G_{k}(r) F_{k^{\prime}}(r) r^{2} d r .
\end{aligned}
$$

The two first integrals are $\left\langle r_{F}\right\rangle$ and $\left\langle r_{G}\right\rangle$, respectively, and the effect of retardation is introduced by the two latter integrals, which expressed as a function of the RR-integrals read as

$$
\begin{aligned}
\int_{0}^{\infty} & F_{k}(r) G_{k^{\prime}}(r) r^{2} d r \\
= & \mathcal{A} f_{1 k} g_{1 k^{\prime}} I_{3}\left(m, 2 v_{k}+1, m^{\prime}, 2 v_{k^{\prime}}+1\right) \\
& +\mathcal{B} f_{1 k} g_{2 k^{\prime}} I_{2}\left(m, 2 v_{k}+1, m^{\prime}+1,2 v_{k^{\prime}}-1\right) \\
& +\mathcal{C} f_{2 k^{\prime}} g_{1 k^{\prime}} I_{2}\left(m+1,2 v_{k}-1, m^{\prime}, 2 v_{k^{\prime}}+1\right) \\
& +\mathcal{D} f_{2 k} g_{2 k^{\prime}} I_{1}\left(m+1,2 v_{k}-1, m^{\prime}+1,2 v_{k^{\prime}}-1\right), \\
\int_{0}^{\infty} & G_{k}(r) F_{k^{\prime}}(r) r^{2} d r=\mathcal{A} g_{1 k} f_{1 k^{\prime}} I_{3}\left(m, 2 v_{k}+1, m^{\prime}, 2 v_{k^{\prime}}+1\right) \\
+ & \mathcal{B} g_{1 k} f_{2 k^{\prime}} I_{2}\left(m, 2 v_{k}+1, m^{\prime}+1,2 v_{k^{\prime}}-1\right) \\
+ & \mathcal{C} g_{2 k} f_{1 k^{\prime}} I_{2}\left(m+1,2 v_{k}-1, m^{\prime}, 2 v_{k^{\prime}}+1\right) \\
+ & \mathcal{D} g_{2 k} f_{2 k^{\prime}} I_{1}\left(m+1,2 v_{k}-1, m^{\prime}+1,2 v_{k^{\prime}}-1\right),
\end{aligned}
$$

This last expression for $r_{k k^{\prime}}$ reproduces the semi-relativistic expression (11) when the lowest-order term $L=1$ in Eq. (31) is considered.

\section{Results}

With the purpose of validating the new expression for matrix elements and to check its results, we begin this section comparing the values obtained from the expression (16) with the ones obtained from numerical solution of the radial component of the wave function. In Table 1 we show the values of matrix elements for some ions of the Ne isoelectronic sequence computed for transitions of a single electron from the ground state $k$ to several excited states $k^{\prime}$. As seen the difference $\Delta r_{k k^{\prime}}$ between values obtained from the new analytical expression and those computed from the wave function integration is about the numerical machine error, indicating that the 
Table 2

RR-integral values $I_{\alpha}$ and matrix elements $r_{k K}$ for Li-like and Be-like iron ions computed for transitions of a single electron from $2 s_{1 / 2}$ to several excited states $k^{\prime}$. Values of screened charges $Q_{k}$ and $Q_{k}$ are also shown together with the arguments and order of the integrals.

\begin{tabular}{|c|c|c|c|c|c|c|c|c|c|c|}
\hline Ion & $k^{\prime}$ & $Q_{k}$ & $Q_{k}$ & $q_{k}$ & $p_{k}$ & $q_{k^{\prime}}$ & $p_{k^{\prime}}$ & $\alpha$ & $I_{\alpha}$ & $r_{k k^{\prime}}$ \\
\hline \multirow[t]{8}{*}{$\mathrm{Fe}^{+23}$} & $3 p_{1 / 2}$ & 24.6253 & 24.1108 & 0 & 2.9674 & 1 & 2.9688 & 2 & $-1.2804 \times 10^{-6}$ & $1.92 \times 10^{-2}$ \\
\hline & & & & 0 & 2.9674 & 2 & 0.9688 & 1 & $3.6774 \times 10^{-6}$ & \\
\hline & & & & 1 & 0.9674 & 1 & 2.9688 & 1 & $3.2301 \times 10^{-5}$ & \\
\hline & & & & 1 & 0.9674 & 2 & 0.9688 & 0 & $-1.0770 \times 10^{-4}$ & \\
\hline & $3 p_{3 / 2}$ & 24.6253 & 24.1076 & 0 & 2.9674 & 0 & 4.9845 & 2 & $5.0234 \times 10^{-7}$ & $1.14 \times 10^{-1}$ \\
\hline & & & & 0 & 2.9674 & 1 & 2.9845 & 1 & $-1.2458 \times 10^{-6}$ & \\
\hline & & & & 1 & 0.9674 & 0 & 4.9845 & 1 & $-9.0461 \times 10^{-6}$ & \\
\hline & & & & 1 & 0.9674 & 1 & 2.9845 & 0 & $3.1661 \times 10^{-5}$ & \\
\hline \multirow[t]{8}{*}{$\mathrm{Fe}^{+22}$} & $3 d_{3 / 2}$ & 23.503 & 22.0437 & 0 & 2.9704 & 0 & 4.987 & 2 & $7.8053 \times 10^{-7}$ & $1.89 \times 10^{-1}$ \\
\hline & & & & 0 & 2.9704 & 1 & 2.987 & 1 & $-1.5117 \times 10^{-6}$ & \\
\hline & & & & 1 & 0.9704 & 0 & 4.987 & 1 & $-1.3488 \times 10^{-5}$ & \\
\hline & & & & 1 & 0.9704 & 1 & 2.987 & 0 & $3.8756 \times 10^{-5}$ & \\
\hline & $4 s_{1 / 2}$ & 23.503 & 22.4893 & 0 & 2.9704 & 2 & 2.9729 & 2 & $-2.7422 \times 10^{-6}$ & $1.25 \times 10^{-2}$ \\
\hline & & & & 0 & 2.9704 & 3 & 0.9729 & 1 & $3.5819 \times 10^{-6}$ & \\
\hline & & & & 1 & 0.9704 & 2 & 2.9729 & 1 & $6.7681 \times 10^{-5}$ & \\
\hline & & & & 1 & 0.9704 & 3 & 0.9729 & 0 & $-1.1069 \times 10^{-4}$ & \\
\hline
\end{tabular}

new analytical expression of matrix elements is exact. The values of screened charges and energies corresponding to initial and final configurations of each transition are also shown. They have been computed making use of the NRSHM [22].

To numerically illustrate the order of magnitude of the RR integrals $I_{\alpha}$, Table 2 shows values involved in the calculation of matrix elements for transitions of one electron from the state $2 s_{1 / 2}$ to different excited states $k^{\prime}$, for Li-like and Be-like iron ions. This table also shows values for screened charges $Q_{k}$ and $Q_{k}$, integral arguments $q_{k}, p_{k}, q_{k^{\prime}}, p_{k}$, and integral order $\alpha$.

In Table 3 we compare absolute values of radial matrix elements for some members of the $\mathrm{Li}$ isoelectronic sequence with Dirac-Hartree-Fock (DHF) values, Dirac-quantumdefect orbitals (DQDO) and Dirac-supersymmetry-based quantum-defect theory (DSQDT) values were taken from Ref. [44]. The values of matrix elements obtained for neutral lithium have a big relative error when comparing with the ones from the other models. This is due to the use of the NRSHM which does not provide good values of screened charges for neutral or slightly ionized elements and it cannot be attributed to the expression presented in this paper. In this model, configurations belonging to neutral atoms and two first ionization states were not included in the fit of the screening constants, since the NRSHM was conceived to compute the atomic properties of ICF plasmas. In this kind of plasmas the abundances of these species are negligible except during the first moments of target irradiation. Moreover, as the ionization state increases in a multielectron atom, the electron-electron term in the Hamiltonian decreases, so the behavior of the system is better modeled by a hydrogenic approximation. In fact, we found out that considering these configurations in the fitting of the screening constants led to worse results for the remaining states of ionization. Accordingly, Table 3 shows that the values of matrix elements improve considerably when increasing the value of $Z$ for the Li-like sequence.
Table 3

Absolute values of radial matrix elements between states $2 s^{2} S$ and $n p$ ${ }^{2} P_{1 / 2,3 / 2}(n=2,3)$ for some members of the Li isoelectronic sequence. DQDO, DSQDT and DHF are fully relativistic results [44]. Values are given in a.u.

\begin{tabular}{llllllr}
\hline lon & Final state & DQDO & DSQDT & DHF & This work & $\varepsilon_{r}^{D H F}(\%)$ \\
\hline $\mathrm{Li}$ & $2 p_{1 / 2}$ & 4.1575 & 4.1169 & 4.1205 & 3.8110 & 7.5112 \\
& $2 p_{3 / 2}$ & 4.1576 & 4.1169 & 4.1205 & 3.8230 & 7.2200 \\
& $3 p_{1 / 2}$ & 0.2238 & 0.2100 & 0.1900 & 0.0193 & 89.8421 \\
& $3 p_{3 / 2}$ & 0.2238 & 0.2100 & 0.1899 & 0.0071 & 96.2612 \\
$\mathrm{Mo}^{+39}$ & $2 p_{1 / 2}$ & 0.1244 & 0.1243 & 0.1241 & 0.1244 & -0.2417 \\
& $2 p_{3 / 2}$ & 0.1271 & 0.1270 & 0.1269 & 0.1272 & -0.2364 \\
& $3 p_{1 / 2}$ & 0.0710 & 0.0711 & 0.0711 & 0.0714 & -0.4219 \\
& $3 p_{3 / 2}$ & 0.0669 & 0.0671 & 0.0670 & 0.0674 & -0.5970 \\
$\mathrm{U}^{+}+89$ & $2 p_{1 / 2}$ & 0.0460 & 0.0460 & 0.0459 & 0.0461 & -0.4357 \\
& $2 p_{3 / 2}$ & 0.0523 & 0.0523 & 0.0522 & 0.0523 & -0.1916 \\
& $3 p_{1 / 2}$ & 0.0293 & 0.0295 & 0.0295 & 0.0296 & -0.3390 \\
& $3 p_{3 / 2}$ & 0.0197 & 0.0199 & 0.0199 & 0.0202 & -1.5075 \\
\hline
\end{tabular}

To illustrate the goodness of the expressions obtained, oscillator strengths for several transitions of Li-like and Na-like ions are presented in Tables 4 and 5. The NRSHM has been used in this work to compute transition energies between levels necessaries for computing these oscillator strengths [41]. Table 4 shows our results for transitions of a single electron from the level $2 s_{1 / 2}$ to $2 p_{1 / 2}, 2 p_{3 / 2}, 3 p_{1 / 2}$ and $3 p_{3 / 2}$ for some Li-like ions. They are compared with results obtained from WKB model, Klein-Gordon WKB model (WKB-KG), self-consistent Hartree-Slater model (CAHS), fully relativistic supersymmetry-inspired quantum-defect model (DSQDT), and with ab initio results, obtained from the compilation published in Ref. [13]. Column labeled NRSHM contains values computed in the semi-relativistic NRSHM approach explained in Section 3, whereas the column labeled NRSHM* shows 
Table 4

Oscillator strengths corresponding to transitions from $2 s_{1 / 2}$ to $2 p_{1 / 2}, 2 p_{3 / 2}, 3 p_{1 / 2}$ and $3 p_{3 / 2}$ respectively for some Li-like ions computed in the semirelativistic approach NRSHM, and the fully relativistic approach NRSHM*.

\begin{tabular}{|c|c|c|c|c|c|c|c|c|c|c|}
\hline $\mathrm{Z}$ & Ion & WKB & $\mathrm{KG}-1^{\mathrm{a}}$ & $\mathrm{KG}-\mathrm{O}^{\mathrm{b}}$ & $\mathrm{KG}-\mathrm{NK}^{\mathrm{c}}$ & $\mathrm{CAHS}^{\mathrm{d}}$ & DSQDT $^{\mathrm{e}}$ & $\mathrm{Ab}$ initio & NRSHM & NRSHM $^{*}$ \\
\hline \multirow[t]{4}{*}{18} & \multirow{4}{*}{$\mathrm{Ar}^{15+}$} & 0.0260 & 0.0261 & 0.0260 & 0.0261 & 0.0260 & 0.0260 & $0.0260^{f}$ & 0.0260 & 0.0260 \\
\hline & & 0.0576 & 0.0578 & 0.0575 & 0.0577 & 0.0575 & 0.0575 & 0.0580 & 0.0606 & 0.0606 \\
\hline & & 0.1225 & 0.1214 & 0.1226 & 0.1217 & 0.1200 & 0.1205 & $0.1200^{f}$ & 0.1209 & 0.1204 \\
\hline & & 0.2450 & 0.2427 & 0.2451 & 0.2433 & 0.2360 & 0.2365 & 0.2360 & 0.2376 & 0.2368 \\
\hline \multirow[t]{4}{*}{20} & \multirow[t]{4}{*}{$\mathrm{Ca}^{17+}$} & 0.0233 & 0.0234 & 0.0232 & 0.0233 & 0.0232 & 0.0232 & $0.0234^{g}$ & 0.0233 & 0.0233 \\
\hline & & 0.0535 & 0.0538 & 0.0534 & 0.0537 & 0.0534 & 0.0534 & 0.0538 & 0.0562 & 0.0562 \\
\hline & & 0.1288 & 0.1274 & 0.1289 & 0.1277 & 0.1223 & 0.1229 & & 0.1234 & 0.1227 \\
\hline & & 0.2516 & 0.2486 & 0.2517 & 0.2494 & 0.2386 & 0.2401 & & 0.2415 & 0.2405 \\
\hline \multirow[t]{4}{*}{22} & \multirow[t]{4}{*}{$\mathrm{Ti}^{19+}$} & 0.0210 & 0.0211 & 0.0209 & 0.0211 & 0.0210 & 0.0210 & $0.0212^{\mathrm{g}}$ & 0.0210 & 0.0210 \\
\hline & & 0.0507 & 0.0509 & 0.0505 & 0.0508 & 0.0506 & 0.0506 & 0.0509 & 0.0530 & 0.0530 \\
\hline & & 0.1311 & 0.1292 & 0.1311 & 0.1297 & 0.1244 & 0.1248 & & 0.1254 & 0.1246 \\
\hline & & 0.2545 & 0.2508 & 0.2546 & 0.2518 & 0.2413 & 0.2426 & & 0.2442 & 0.2431 \\
\hline \multirow[t]{4}{*}{24} & \multirow{4}{*}{$\mathrm{Cr}^{21+}$} & 0.0192 & 0.0193 & 0.0191 & 0.0193 & 0.0192 & 0.0192 & $0.0193^{\mathrm{g}}$ & 0.0192 & 0.0192 \\
\hline & & 0.0488 & 0.0491 & 0.0486 & 0.0490 & 0.0487 & 0.0487 & 0.0490 & 0.0509 & 0.0509 \\
\hline & & 0.1331 & 0.1308 & 0.1331 & 0.1314 & 0.1261 & 0.1265 & & 0.1271 & 0.1260 \\
\hline & & 0.2565 & 0.2520 & 0.2566 & 0.2532 & 0.2430 & 0.2444 & & 0.2462 & 0.2448 \\
\hline \multirow[t]{4}{*}{26} & \multirow[t]{4}{*}{$\mathrm{Fe}^{23+}$} & 0.0177 & 0.0178 & 0.0176 & 0.0177 & 0.0177 & 0.0177 & $0.0180^{f}$ & 0.0176 & 0.0176 \\
\hline & & 0.0477 & 0.0481 & 0.0474 & 0.0479 & 0.0476 & 0.0476 & 0.0480 & 0.0495 & 0.0495 \\
\hline & & 0.1353 & 0.1326 & 0.1354 & 0.1333 & 0.1280 & 0.1277 & $0.1280^{f}$ & 0.1285 & 0.1272 \\
\hline & & 0.2706 & 0.2651 & 0.2707 & 0.2666 & 0.2430 & 0.2453 & 0.2450 & 0.2474 & 0.2457 \\
\hline \multirow[t]{4}{*}{36} & \multirow[t]{4}{*}{$\mathrm{Kr}^{33+}$} & 0.0127 & 0.0129 & 0.0126 & 0.0128 & 0.0127 & 0.0127 & $0.0127^{f}$ & 0.0125 & 0.0125 \\
\hline & & 0.0502 & 0.0510 & 0.0496 & 0.0506 & 0.0500 & 0.0498 & 0.0499 & 0.0508 & 0.0509 \\
\hline & & 0.1398 & 0.1341 & 0.1400 & 0.1357 & 0.1320 & 0.1322 & $0.1350^{\mathrm{h}}$ & 0.1328 & 0.1302 \\
\hline & & 0.2575 & 0.2466 & 0.2578 & 0.2497 & 0.2430 & 0.2438 & 0.2490 & 0.2464 & 0.2431 \\
\hline \multirow[t]{4}{*}{42} & \multirow{4}{*}{$\mathrm{Mo}^{39+}$} & 0.0109 & 0.0112 & 0.0107 & 0.0111 & 0.0109 & 0.0109 & 0.0110 & 0.0107 & 0.0106 \\
\hline & & 0.0563 & 0.0575 & 0.0554 & 0.0569 & 0.0561 & 0.0556 & 0.0560 & 0.0563 & 0.0563 \\
\hline & & 0.1424 & 0.1343 & 0.1425 & 0.1366 & 0.1330 & 0.1336 & 0.1330 & 0.1342 & 0.1307 \\
\hline & & 0.2529 & 0.2376 & 0.2534 & 0.2421 & 0.2380 & 0.2384 & 0.2380 & 0.2419 & 0.2374 \\
\hline
\end{tabular}

${ }^{a} \mathrm{KG}-1$ : Lagmago Kamta et al. [13].

${ }^{\mathrm{b}} \mathrm{KG}-0$ : Lagmago Kamta et al. [13].

' KG-NK: Nana Engo et al. [16].

d CAHS: Theodosiou et al. [46].

e DSQDT: Nana Engo et al. [18].

${ }^{f}$ DHF: Armstrong et al. [47].

g MCDF: Cheng et al. [48].

${ }^{\text {h }}$ Quasirelativistic RHF: Karwowski and Szulkin [49].

Table 5

Experimental and theoretical oscillator strengths corresponding to transitions from $3 s_{1 / 2}$ to $3 p_{1 / 2}$, first entry, and $3 p_{3 / 2}$, second entry, for some Na-like ions computed with the fully relativistic approach. Table compiled from Ref. [45].

\begin{tabular}{lllll}
\hline Ion & ANDC & NRSHM $^{*}$ & Other experiment & Theory \\
\hline $\mathrm{Ti}^{11+}$ & $0.143 \pm 0.009$ & 0.146 & & 0.156 \\
& $0.312 \pm 0.019$ & 0.336 & & 0.322 \\
$\mathrm{Fe}^{15+}$ & $0.115 \pm 0.007$ & 0.123 & $0.124^{\mathrm{b}}$ & 0.125 \\
& $0.244 \pm 0.015$ & 0.289 & $0.244^{\mathrm{c}}$ & 0.272 \\
$\mathrm{Ni}^{17+}$ & $0.109 \pm 0.007$ & 0.114 & $0.114^{\mathrm{d}}$ & 0.115 \\
& $0.226 \pm 0.014$ & 0.270 & $0.237^{\mathrm{d}}$ & 0.255 \\
$\mathrm{Cu}^{18+}$ & $0.112 \pm 0.007$ & 0.110 & $0.112^{\mathrm{e}}$ & 0.111 \\
\hline
\end{tabular}

${ }^{\text {a }} \mathrm{Kim}$ and Cheng [50], relativistic Hartree-Fock. Values for Ti, Ni, and $\mathrm{Cu}$ are interpolated.

${ }^{\mathrm{b}}$ Buchet et al. [51], multiexponential fit.

' Buchet et al. [51], ANDC.

${ }^{d}$ Pegg et al. [52].

e Pegg et al. [53]. the same values computed using the fully relativistic calculation of matrix elements discussed in Section 5.1. In the calculations presented here the convergence was reached taking $N=4$ in Eq. (27). There is a good agreement with the reference values for the results obtained with both approaches, being significantly better when they are calculated in the fully relativistic approach. Thus, the relative errors of the oscillator strengths for the transitions shown, computed in the semi-relativistic approximation, have an average error of $1.59 \%$ in relation to CASH values, and $1.45 \%$ compared with those obtained by the DSQDT method. These values are generally lower when using the fully relativistic approach, decreasing in both cases around $0.1 \%$. In any case the error never exceeds the $6 \%$, these results being similar to the difference between the models considered.

Finally, Table 5 shows oscillator strengths for transitions from $3 s_{1 / 2}$ to $3 p_{1 / 2}$ and $3 p_{3 / 2}$ of Na-like ions calculated in the fully relativistic approximation and compared with experimental and theoretical results [45]. Again, the 
oscillator strengths obtained with the NRSHM are in good agreement with the experimental ones.

\section{Conclusions}

We have developed an analytical expression to determine the values of relativistic matrix elements in both semirelativistic and fully relativistic approaches making use of relativistic screened hydrogenic wave functions. To obtain this formula has been necessary to determine analytically the value of radial integrals expressions of the relativistic Laguerre polynomials that depend on the screened charge of each of the levels involved in a transition. The final formula is expressed in terms of a sole Gauss hypergeometric function and it has been tested which gives exact values when comparing with those obtained by means of the numerical integration of the radial matrix elements using relativistic screened hydrogenic wavefunctions.

In order to illustrate the usefulness of the new expression of relativistic matrix elements in the computation of the optical properties of the atoms, it has been used together with the NRSHM to obtain the oscillator strengths for $\mathrm{Li}$ - and Na-like ions. Comparisons with values obtained from more sophisticated models and experimental data indicate that it is valid for the description of medium and highly ionized atoms such as those typically found in plasma physics and astrophysics. We would like to remark that the expressions for matrix elements obtained in this paper have been derived in the frame of the validity of the relativistic screened hydrogenic model, so that they should be used with caution outside of this scope.

\section{Acknowledgments}

This work has been partially supported by a Research Project of the Spanish Ministry of Science and Innovation (ENE2009-11208), by a Research Project of the Agencia Canaria de Investigación, Innovación y Sociedad de la Información of the regional government of the Canary Islands (SOLSUBC2008000057) and also by the Keep-in Touch project of the European Union.

\section{Appendix A. Alternative method to solve the RR-Integral}

\section{A.1. Analytical expression of the RR-Integral}

In this section we will show an alternative method to obtain an analytical expression for the integral function defined in (14). We start from the general expression

$$
\begin{aligned}
& I_{s}\left(q_{k}, p_{k}, q_{k^{\prime}}, p_{k^{\prime}}\right) \\
& \quad=\int_{0}^{\infty} r^{s} e^{\left(a_{k}+a_{k^{\prime}}\right) r} L_{q_{k}}^{p_{k}}\left(2 a_{k} r\right) L_{q_{k^{\prime}}}^{p_{k^{\prime}}}\left(2 a_{k^{\prime}} r\right) d r, \quad a_{k}+a_{k^{\prime}}>0, s>0
\end{aligned}
$$

The method we are going to describe uses as starting point the generatrix function of Laguerre polynomials [43], which, using the notation defined in this work, can be written as

$$
(1-y)^{-p_{k}-1} e^{\left(-2 a_{k} y /(1-y)\right) r}=\sum_{\mu=0}^{\infty} y^{\mu} L_{\mu}^{p_{k}}\left(2 a_{k} r\right) \quad|y|<1
$$

On multiplying both sides of this equation by the factor $e^{\left[-\left(a_{k}+a_{k^{\prime}}\right) r\right]} r^{s} L_{q_{k^{\prime}}}^{p_{k^{\prime}}}\left(2 a_{k^{\prime}} r\right)$, and integrating from zero to infinity, we obtain

$$
\begin{aligned}
& (1-y)^{-p_{k}-1} \int_{0}^{\infty} e^{-\left(a_{k}+a_{k^{\prime}}+2 a_{k} y /(1-y)\right) r} r^{s} L_{q_{k^{\prime}}}^{p_{k^{\prime}}}\left(2 a_{k^{\prime}} r\right) d r \\
& \quad \times \sum_{\mu=0}^{\infty} y^{\mu} \int_{0}^{\infty} e^{-\left(a_{k}+a_{k^{\prime}}\right) r} r^{s} L_{q_{k^{\prime}}}^{p_{k^{\prime}}}\left(2 a_{k^{\prime}} r\right) L_{\mu}^{p_{k}}\left(2 a_{k} r\right) d r \\
& =\sum_{\mu=0}^{\infty} y^{\mu} I_{S}\left(\mu, p_{k}, q_{k^{\prime}}, p_{k^{\prime}}\right)
\end{aligned}
$$

In the first term of Eq. (A.3) we can express the Laguerre polynomials in terms of confluent hypergeometric function

$L_{q_{k^{\prime}}}^{p_{k^{\prime}}}\left(2 a_{k^{\prime}} r\right)=\frac{\Gamma\left(q_{k^{\prime}}+p_{k^{\prime}}+1\right)}{q_{k^{\prime}} ! \Gamma\left(p_{k^{\prime}}+1\right)} F\left(-q_{k^{\prime}}, p_{k^{\prime}}+1,2 a_{k^{\prime}} r\right)$,

and we can substitute the exponent by

$\left(a_{k}+a_{k^{\prime}}+\frac{2 a_{k} y}{1-y}\right) r=\frac{\left(a_{k}+a_{k^{\prime}}\right)\left(1+a_{k k^{\prime}} y\right)}{1-y} r$,

where $a_{k k^{\prime}}=\left(a_{k}-a_{k^{\prime}}\right) /\left(a_{k}+a_{k^{\prime}}\right)$.

After substituting and rearranging, we obtain for the first term of Eq. (A.3) the final form

$$
\begin{aligned}
& (1-y)^{-p_{k}-1} \frac{\Gamma\left(q_{k^{\prime}}+p_{k^{\prime}}+1\right)}{q_{k^{\prime}} ! \Gamma\left(p_{k^{\prime}}+1\right)} \\
& \quad \times \int_{0}^{\infty} e^{\left[-\left(\left(a_{k}+a_{k^{\prime}}\right)\left(1+a_{k^{\prime}} y\right)\right) /(1-y) r\right]_{r^{s}} F\left(-q_{k^{\prime}}, p_{k^{\prime}}+1,2 a_{k^{\prime}} r\right) d r} \\
& =\frac{\Gamma\left(q_{k}+p_{k^{\prime}}+1\right) \Gamma(s+1)}{q_{k^{\prime}} ! \Gamma\left(p_{k^{\prime}}+1\right)} \frac{(1-y)^{s-p_{k}}}{\left(a_{k}+a_{k^{\prime}}\right)^{s+1}\left(1+a_{k k^{\prime}} y\right)^{s+1}} \\
& \quad \times{ }_{2} F_{1}\left[-q_{k^{\prime}}, s+1, p_{k^{\prime}}+1, \frac{\left(1-a_{k k^{\prime}}\right)(1-y)}{1+a_{k k^{\prime}} y}\right] \\
& =\frac{\Gamma\left(q_{k^{\prime}}+p_{k^{\prime}}+1\right) \Gamma(s+1)}{q_{k^{\prime}} ! \Gamma\left(p_{k}+1\right)} f(y),
\end{aligned}
$$

where ${ }_{2} F_{1}$ is the hypergeometric function and $f(y)$ has the form

$$
\begin{aligned}
f(y) & =\frac{(1-y)^{s-p_{k}}}{\left(a_{k}+a_{k^{\prime}}\right)^{s+1}\left(1+a_{k k^{\prime}} y\right)^{s+1}}{ }_{2} F_{1} \\
& \times\left[-q_{k^{\prime}}, s+1, p_{k^{\prime}}+1, \frac{\left(1-a_{k k^{\prime}}\right)(1-y)}{1+a_{k k^{\prime}} y}\right] .
\end{aligned}
$$

Thus Eq. (A.3) can be expressed as

$$
\sum_{\mu=0}^{\infty} y^{\mu} I_{s}\left(\mu, p_{k}, q_{k^{\prime}}, p_{k^{\prime}}\right)=\frac{\Gamma\left(q_{k^{\prime}}+p_{k^{\prime}}+1\right) \Gamma(s+1)}{q_{k^{\prime}} ! \Gamma\left(p_{k^{\prime}}+1\right)} f(y) \text {. }
$$

Taking the series expansion of $f(y)$ around the origin, this equation turns into

$$
\begin{aligned}
& \sum_{\mu=0}^{\infty} y^{\mu} I_{s}\left(\mu, p_{k}, q_{k^{\prime}}, p_{k^{\prime}}\right)=\frac{\Gamma\left(q_{k^{\prime}}+p_{k^{\prime}}+1\right) \Gamma(s+1)}{q_{k} ! \Gamma\left(p_{k}+1\right)} \\
& \times\left[f(0)+\frac{f^{(1)}(0)}{1 !} y+\frac{f^{(2)}(0)}{2 !} y^{2}+\cdots+\frac{f^{(\mu)}(0)}{\mu !} y^{\mu}\right] .
\end{aligned}
$$

Therefore, identifying the general terms of the members of the above equality and choosing $\mu=q_{k}$, the integral 
(17) is of the form

$I_{s}\left(q_{k}, p_{k}, q_{k^{\prime}}, p_{k^{\prime}}\right)=\frac{\Gamma\left(q_{k^{\prime}}+p_{k^{\prime}}+1\right) \Gamma(s+1)}{q_{k} ! q_{k^{\prime}} ! \Gamma\left(p_{k^{\prime}}+1\right)} f^{\left(q_{k}\right)}(0)$,

where $f^{\left(q_{k}\right)}(0)$ is the derivative of order $q_{k}$ at the origin of the function $f(y)$.

\section{A.2. Exact calculation of $\mathbf{f}^{\left(\mathbf{q}_{\mathbf{k}}\right)}(\mathbf{0})$ by substituting series}

In this section we explain how to obtain the derivatives in Eq. (A.6) accurately. The method consists in taking the series expansions of each of the terms that form a function and making the composition throughout the sum, the product, the division or the power of the partials series obtained. The aim is to find the derivative of order $q_{k}$ of the function $f(y)$, which is equivalent to calculate the coefficient of the Taylor series expansion at the origin with the same order. Due to the fact that $f(y)$ is an extremely complicated function, the direct calculation of the derivative is not an abordable task, so we will split the problem into simpler parts and we will construct the final series making use of the processes mentioned above.

The first step is to develop the hypergeometric function ${ }_{2} F_{1}$ in Eq. (A.4)

$f(y)=\frac{(1-y)^{s-p_{k}}}{\left(a_{k}+a_{k^{\prime}}\right)^{s+1}\left(1+a_{k k^{\prime}} y\right)^{s+1}} \sum_{i=1}^{q_{k^{\prime}}} c_{i} z^{i}$

where it has been defined $z$ as

$z=\frac{\left(1-a_{k k}\right)(1-y)}{1+a_{k k} y}$

In this equation, coefficients $c_{i}$ can be obtained by the recurrence relation of the hypergeometric series

$c_{0}=1, \quad \frac{c_{i+1}}{c_{i}}=\frac{\left(-q_{k^{\prime}}+i\right)(s+i+1)}{\left(p_{k^{\prime}}+i+1\right)(i+1)}$

Rearranging the terms, we obtain for $f(y)$ the following expression:

$$
\begin{aligned}
f(y) & =\frac{1}{\left(a_{k}+a_{k^{\prime}}\right)^{s+1}} \sum_{i=1}^{q_{k^{\prime}}} c_{i}\left[\frac{(1-y)^{s-p_{k}}}{\left(1+a_{k k^{\prime}} y\right)^{s+1}}\right] z^{i} \\
& =\frac{1}{\left(a_{k}+a_{k}\right)^{s+1}} \sum_{i=1}^{q_{k^{\prime}}} c_{i}\left(1-a_{k k}\right)^{i}\left[\frac{(1-y)^{s+i-p_{k}}}{\left(1+a_{k k} y\right)^{s+i+1}}\right]
\end{aligned}
$$

If we expand in power series the last term in brackets and after some tedious manipulations, the function $f(y)$ reads as

$$
\begin{aligned}
f(y) & =\frac{1}{\left(a_{k}+a_{k^{\prime}}\right)^{s+1}} \sum_{i=1}^{q_{k^{\prime}}} c_{i} \sum_{t=0}^{\infty} d_{t}^{i} y^{t} \\
& =\sum_{t=0}^{\infty}\left(\frac{t !}{\left(a_{k}+a_{k^{\prime}}\right)^{s+1}} \sum_{i=1}^{q_{k^{\prime}}} c_{i} d_{t}^{i}\right) \frac{y^{t}}{t !},
\end{aligned}
$$

where

$d_{t}^{i}=(-1)^{t+1}\left(1-a_{k k^{\prime}}\right)^{i} \sum_{\lambda=0}^{t} b_{\lambda}$

and where the coefficients $b_{\lambda}$ can be expressed as

$b_{0}=\frac{\Gamma\left(i+s+1-p_{k}\right)}{\Gamma\left(i+s+1-p_{k}-q_{k}\right) q_{k} !}$,

$$
\frac{b_{\lambda+1}}{b_{\lambda}}=\frac{a_{k k^{\prime}}\left(q_{k}-\lambda\right)(\lambda+i+s+1)}{(\lambda+1)\left(\lambda+i+s+1-p_{k}-q_{k}\right)}
$$

The derivative of order $q_{k}$ at the origin is then obtained retaining only the coefficients corresponding to $t=q_{k}$ in Eq. (A.11)

$f^{\left(q_{k}\right)}(0)=\frac{q_{k} !}{\left(a_{k}+a_{k^{\prime}}\right)^{s+1}} \sum_{i=0}^{q_{k}} c_{i} d_{q_{k}}^{i}$.

Finally, the expression for the RR-integrals (A.6) reads as $I_{s}\left(q_{k}, p_{k}, q_{k}, p_{k^{\prime}}\right)=\frac{\Gamma\left(q_{k^{\prime}}+p_{k^{\prime}}+1\right) \Gamma(s+1)}{q_{k^{\prime}} ! \Gamma\left(p_{k^{\prime}}+1\right)\left(a_{k}+a_{k^{\prime}}\right)^{s+1}} \sum_{i=0}^{q_{k^{\prime}}} c_{i} d_{q_{k}}^{i}$

\section{References}

[1] Badawi M, Bessis N, Bessis $G$, Hadinger $G$. Closed-form hydrogenic radial $r^{k}$ matrix elements and the factorization method. Phys Rev A 1973;8:727-33.

[2] Dong S, Chen C, Lozada-Cassou M. Some recurrence relations among the radial matrix elements for the relativistic hydrogenic atoms. Phys Lett A 2004:333(3-4):193-203.

[3] D'yachkov LG, Pankratov PM. Semiclassical formula for radial integrals of bound-bound dipole transitions. J Phys B: At Mol Opt Phys $1991 ; 24(9): 2267$.

[4] D'yachkov LG, Pankratov PM. On the use of the semiclassical approximation for the calculation of oscillator strengths and photoionization cross sections. J Phys B: At Mol Opt Phys 1994;27(3):461.

[5] Kaulakys B. Consistent analytical approach for the quasi-classical radial dipole matrix elements. J Phys B: At Mol Opt Phys $1995 ; 28(23): 4963$

[6] Kostelecký VA, Nieto MM. Analytical wave functions for atomic quantum-defect theory. Phys Rev A 1985;32:3243-6.

[7] More R, Warren K. Semiclassical calculation of matrix elements. Ann Phys 1991;207(2):282-342.

[8] Pankratov P, Meyer-ter Vehn J. Semiclassical description of dipole matrix elements for arbitrary $n l \rightarrow n l^{\prime}$ transitions in nonhydrogenic ions. Phys Rev A 1992;46:5500-5.

[9] Picart J, de Izarra C, Oumarou B, Tran Minh N, Klarsfeld S. Recurrence relations for multipole radial integrals in the semiclassical coulomb approximation. Phys Rev A 1991;43:2535-7.

[10] Sánchez ML, Moreno B, López Piñeiro A. Matrix-element calculations for hydrogenlike atoms. Phys Rev A 1992;46:6908-13.

[11] Ilarraza-Lomelí AC, Valdés-Martínez MN, Salas-Brito AL, Martínez y Romero RP, Núñez Yépez HN. Calculation of matrix elements in relativistic quantum mechanics. Int J Quantum Chem 2002;90(1): 195-201.

[12] Kwato Njock MG, Lagmago Kamta G, Nana Engo SG, Oumarou B, Ntamack GE. Good's quasiclassical dipole matrix elements for discrete states in nonhydrogenic ions. Phys Lett A 1998;243(1-2): 52-9.

[13] Lagmago Kamta G, Nana Engo SG, Kwato Njock MG, Oumarou B. Consistent description of Klein-Gordon dipole matrix elements. J Phys B: At Mol Opt Phys 1998:31(5):963

[14] Martínez y Romero RP, Núñez Yépez HN, Salas-Brito AL. Relativistically extended Blanchard recurrence relation for hydrogenic matrix elements. J Phys B: At Mol Opt Phys 2001;34(7):1261.

[15] More RM. Relativistic semiclassical atomic transition rates. J Quant Spectrosc Radiat Transfer 1994;51(1-2):237-46.

[16] Nana Engo SG, Owono Owono LC, Dada JP, Waha Ndeuna L, Kwato Njock MG, Oumarou B, et al. Relativistic semiclassical description of dipole matrix elements for arbitrary nlj to n'l'j' transitions in non-hydrogenic ions. J Phys B: At Mol Opt Phys 1995;28(12):2333.

[17] Davydkin VA, Osyannov VD, Zon BA. Oscillator strengths, polarizabilities, and hyperpolarizabilities of Rydberg states. Laser Phys 1993;3:449-61.

[18] Nana Engo SG, Kwato Njock MG, Owono Owono LC, Oumarou B, Lagmago Kamta $G$, Motapon 0 . Comparison of phenomenological and supersymmetry-inspired quantum-defect methods in their relativistic and quasirelativistic formulations. Phys Rev A 1997;56: 2624-47. 
[19] Faussurier G, Blancard C, Decoster A. New screening coefficients for the hydrogenic ion model including l-splitting for fast calculations of atomic structure in plasmas. J Quant Spectrosc Radiat Transfer 1997;58:233-60.

[20] Layzer D. On a screening theory of atomic spectra. Ann Phys 1959;8:271-96

[21] Mayer H. Methods of opacity calculations. Tech. Rep. (Report LA647, Los Alamos Scientific Laboratory, Los Alamos, NM); 1947.

[22] Mendoza MA, Rubiano JG, Gil JM, Rodríguez R, Florido R, Martel P, et al. A new set of relativistic screening constants for the screened hydrogenic model. High Energy Density Phys 2011;7(3):169-79.

[23] More R. Electronic energy-levels in dense plasmas. J Quant Spectrosc Radiat Transfer 1982;27:345-57.

[24] Rubiano JG, Rodríguez R, Gil JM, Ruano FH, Martel P, Mínguez E. A screened hydrogenic model using analytical potentials. I Quant Spectrosc Radiat Transfer 2002;72(5):575-88.

[25] Slater JC. Atomic shielding constants. Phys Rev 1930;36(1):57-64.

[26] Bel'kov SA, Bondarenko SV, Mitrofanov EI. Equation of matter state in the average-ion model for a laser-produced plasma. Quantum Electron 2000;30:963-9.

[27] Chung HK, Chen M, Morgan W, Ralchenko Y, Lee R. Flychk: generalized population kinetics and spectral model for rapid spectroscopic analysis for all elements. High Energy Density Phys $2005 ; 1(1): 3-12$.

[28] Eidmann K. Radiation transport and atomic physics modeling in high-energy-density laser-produced plasmas. Laser Part Beams $1994 ; 12(02): 223-44$

[29] Faussurier G. Superconfiguration accounting approach versus average-atom model in local-thermodynamic-equilibrium highly ionized plasmas. Phys Rev E 1999;59:7096-109.

[30] Faussurier G, Blancard C, Berthier E. Nonlocal thermodynamic equilibrium self-consistent average-atom model for plasma physics. Phys Rev E 2001;63:026401.

[31] Faussurier G, Blancard C, Renaudin P. Equation of state of dense plasmas using a screened-hydrogenic model with 1-splitting. High Energy Density Phys 2008;4(3-4):114-23.

[32] Lokke WA, Grassberger WH. unpublished UCRL-5227; LLNL; 1977.

[33] Porcherot Q Faussurier G, Blancard C. Thermodynamic conditions of shock Hugoniot curves in hot dense matter. High Energy Density Phys 2010;6(1):76-83.

[34] Rubiano JG, Florido R, Rodríguez R, Gil J, Martel P, Mínguez E. Calculation of the radiative opacity of laser-produced plasmas using a relativistic-screened hydrogenic model. J Quant Spectrosc Radiat Transfer 2004;83(2):159-82.

[35] Tsakiris G, Eidmann K. An approximate method for calculating Planck and Rosseland mean opacities in hot, dense plasmas. J Quant Spectrosc Radiat Transfer 1987;38(5):353-68.

[36] Naqvi AM. Calculations and applications of screened hydrogenic wave functions. J Quant Spectrosc Radiat Transfer 1964;4(5): 597-615.
[37] Khandelwal GS, Pritchard WM, Grubb G, Khan F. Screened hydrogenic radial integrals. Phys Rev A 1989;39:3960-3.

[38] Nikiforov AF, Novikov VG, Solomyannaya AD. Analytical wave functions in self-consistent field models for high-temperature plasma. Laser Part Beams 1996;14(04):765-79.

[39] Upcraft L. The screened hydrogenic model: analytic formulae for matrix elements of radiative and collisional rates in complex ions. High Energy Density Phys 2010;6(3):332-44.

[40] Berestetskii VB, Lifshitz EM, Pitaevski LP. Relativistic quantum theory. Oxford: Pergamon; 1971.

[41] Nikiforov AF, Novikov VG, Uvarov VB, lacob A. Quantum-statistical models of hot dense matter: methods for computation opacity and equation of state. 3764321830; Birkhäuser Basel; 2005.

[42] Sampson DH, Zhang HL, Fontes CJ. A fully relativistic approach for calculating atomic data for highly charged ions. Phys. Rep. 2009;477(4-6):111-214.

[43] Gradshteyn I, Ryzhik 1. Table of integrals, series and products. Elsevier; 2007.

[44] Owono Owono LC, Owona Angue MLC, Kwato Njock MG, Oumarou B. Relativistic atomic matrix elements of $r^{q}$ for arbitrary states in the quantum-defect approximation. Phys Lett A 2004;328(4-5): 341-9.

[45] Hutton R, Engström L, Träbert E. Observation of a discrepancy between experimentally determined atomic lifetimes and relativistic predictions for highly ionized members of the $\mathrm{Na} I$ isoelectronic sequence. Phys Rev Lett 1988;60:2469-72.

[46] Theodosiou CE, Curtis LJ, El-Mekki M. Calculations of $2 p$ lifetimes in the Li sequence. Phys Rev A 1991;44:7144-57.

[47] Armstrong L, Fielder WR, Lin DL. Relativistic effects on transition probabilities in the $\mathrm{Li}$ and Be isoelectronic sequences. Phys Rev A 1976;14:1114-28.

[48] Cheng KT, Kim YK, Desclaux JP. Electric dipole, quadrupole, and magnetic dipole transition probabilities of ions isoelectronic to the first-row atoms, Li through F. At Data Nucl Data Tables 1979;24: 111 .

[49] Karwowski J, Szulkin M. Relativistic calculations on the alkali atoms by a modified Hartree-Fock method. J Phys B: At Mol Phys 1981;14(12): 1915 .

[50] Kim YK, Cheng KT. Transition probabilities for the resonance transitions of Na-like ions. J Opt Soc Am 1978;68:836.

[51] Buchet JP, Buchet-Poulizac MC, Denis A, Désesquelles J, Druetta M. Measurement of radiative lifetimes of low-lying excited states in sodiumlike, fluorinelike, and oxygenlike iron. Phys Rev A 1980;22: 2061-8.

[52] Pegg DJ, Griffin PM, Johnson BM, Jones KW, Kruse TH. A measurement of oscillator strengths for solar XUV flare lines in Ni XVIII. Astrophys J 1978;224:1056-8.

[53] Pegg DJ, Griffin PM, Johnson BM, Jones KW, Cecchi JL, Kruse TH. Radiative lifetimes of the low-lying levels of Na-like copper. Phys Rev A $1977 ; 16: 2008-10$. 